\title{
MEASUREMENT OF ELECTRICAL RESISTIVITY OF NANOSTRUCTURED PLATINUM THIN FILMS AND QUANTUM MECHANICAL ESTIMATES
}

\author{
M. C. Salvadori, A. R.Vaz, R. J. C. Farias and M. Cattani \\ Institute of Physics, University of São Paulo, \\ C.P. 66318, CEP: 05315-970, São Paulo, SP, Brazil, mcsalvadori@if.usp.br
}

Keywords: thin films, electrical resistivity, nanostructured Platinum thin films.

\begin{abstract}
Nanostructured platinum thin films, with thickness going from 1.31 up to $11.66 \mathrm{~nm}$, have been deposited by vacuum arc plasma. We have measured their electrical resistivity and surface roughness. The resistivity results are compared with theoretical predictions obtained using a quantum mechanical approach.
\end{abstract}

\section{Introduction}

As the dimensions of microelectronic circuits and microdevices continue to decrease, the study of the electronic properties of the consisting materials should be addressed in the nanometer scale. In particular, it is of great importance to determine the electronic transport properties of metallic and semiconductor thin films. Since long time ago, considerable efforts have been devoted to study, for instance, the electrical conductivity $\sigma(d)$ of thin films as a function of its thickness $d$, from the experimental and theoretical points of view[1-9].

In this paper, we fabricated platinum thin films, with vacuum arc plasma deposition, with thickness $d$ going from $1.31 \mathrm{~nm}$ up $11.66 \mathrm{~nm}$ and we measured the resistivity $\rho(d)$, the roughness $\Delta(d)$ and determined the surface lateral correlation length $\xi(d)$. Our experimental results are compared with theoretical predictions using the quantum mechanical approach proposed by Fishman and Calecki[3,4].

\section{Experimental Techniques and Results}

The platinum films were deposited by "metal plasma immersion ion implantation and deposition" (MePIIID)[10,11]. The parameters used for the deposition were: 200 A for the arc current, with $5 \mathrm{~ms}$ for arc duration and the frequency of the pulses was $1 \mathrm{~Hz}$. The substrate used for the platinum film deposition was ordinary glass microscope slide. In our precedent works[12,13] Pt thin films have been deposited by the same technique; there are clearly shown the nanostructured nature of these films and the moundlike structure of their surfaces, due to the Pt grains.

The films thicknesses were evaluated using the deposition rate for the specific system geometry. To measure the deposition rate, a small piece of silicon with an ink mark was located in the substrate holder and 457 pulses were shot. After the deposition, the ink was removed and the step was measured by atomic force microscopy (AFM)[12].

For the resistivity measurement, the electric contacts were made with two aluminum foil stripes glued by colloidal silver on the glass substrate. The area of the substrate, used for the resistivity measurement, has dimensions: $(25.40 \pm 0.05) \mathrm{mm}$ by $(20.0 \pm 0.5) \mathrm{mm}$. This last value is the distance between the contacts. The contacts were then connected to a feed-through, so as to allow the resistance measurement of the platinum film in vacuum. After every couple of shots, the resistance of the film was measured with an ohmmeter. Before each resistance measurement, we have been waited for about 10 seconds to stabilize the system. The resistance measurements were converted to resistivity using the film thickness. 
A separated set of samples was used to measure the roughness of the platinum films in function of the thickness. Nine platinum films were deposited with thickness between $1.0 \mathrm{~nm}$ and $26.6 \mathrm{~nm}$, on glass substrates. The roughness (rms) of each film was measured by scanning tunneling microscopy (STM)[13,14], using a NanoScope IIIA, from Digital. The glass roughness was measured using atomic force microscopy (AFM). The images used for the roughness measurement were $(1 \times 1) \mu \mathrm{m}^{2}$ or $(0.5 \times 0.5) \mu \mathrm{m}^{2}$, with $(512 \times 512)$ pixels. For the STM images we have used commercial Platinum-Iridium tips and for the AFM images we have used commercial silicon nitride tip.

To measure the deposition rate, for the specific system geometry used for the platinum deposition, a sample was prepared with 457 pulses and the film thickness measured by AFM was $(28.6 \pm 0.5) \mathrm{nm}$. With this result, the average deposition rate was $0.0625 \mathrm{~nm}$ per pulse.

The ratio of the measured resistivity $\rho_{\exp }(d)$ by the bulk Pt resistivity $\rho_{\text {bulk }}$, as a function of $d$, is presented in Fig.(1), where the open circles represent the experimental values. The errors for the resistivity ratios are about $2 \sim 3 \%$ and for the thickness, about $0.5 \sim 1 \%$. Our experimental results are quite similar to those recently found by Avrekh et al.[15] The solid line represents the theoretical predictions, calculated in Section 3.

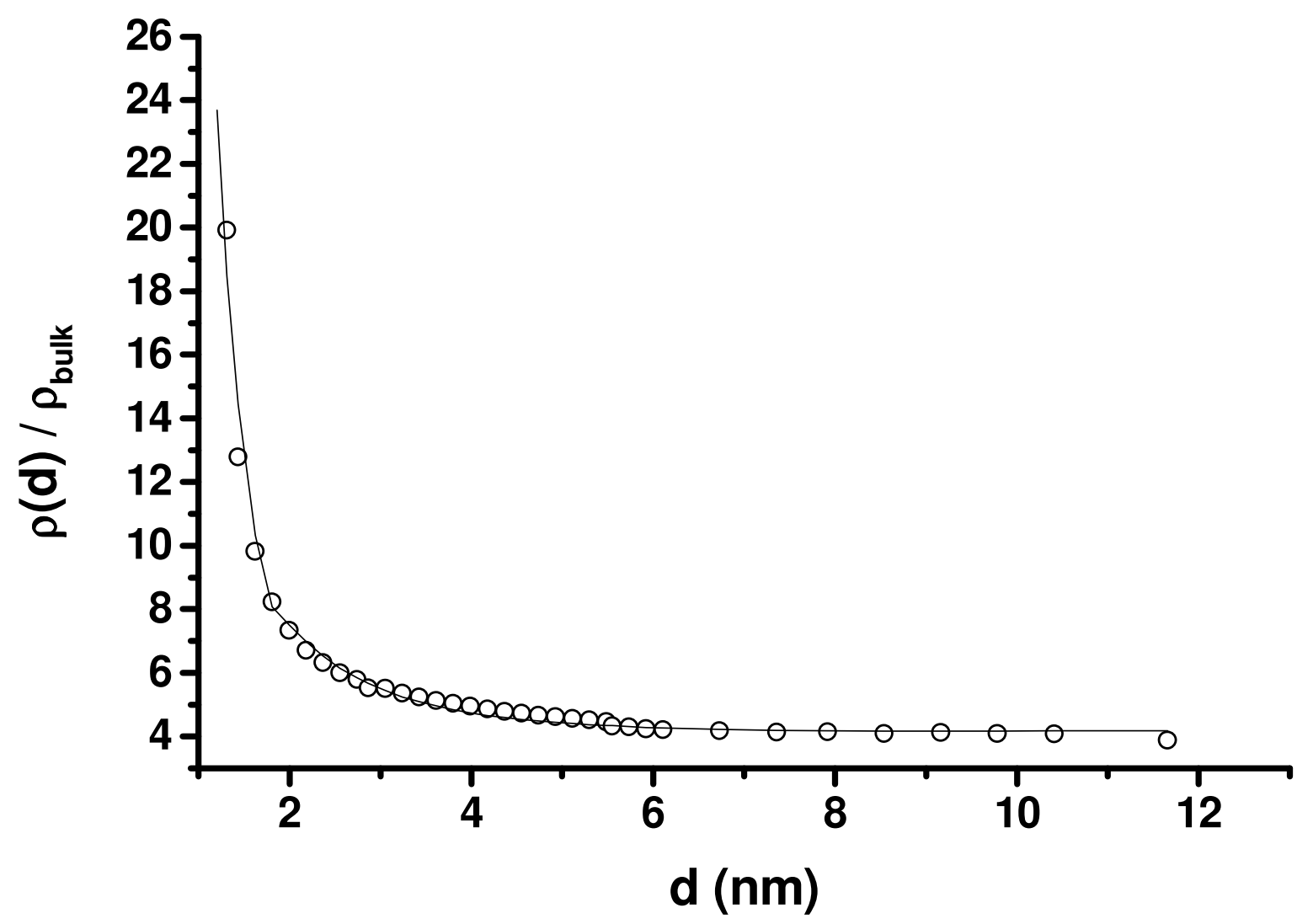

Figure 1: The ratio $\rho_{\exp }(d) / \rho_{\text {bulk }}$ as function of the thickness $d$. The experimental points are represented by open circles and the theoretical results by a solid line. 


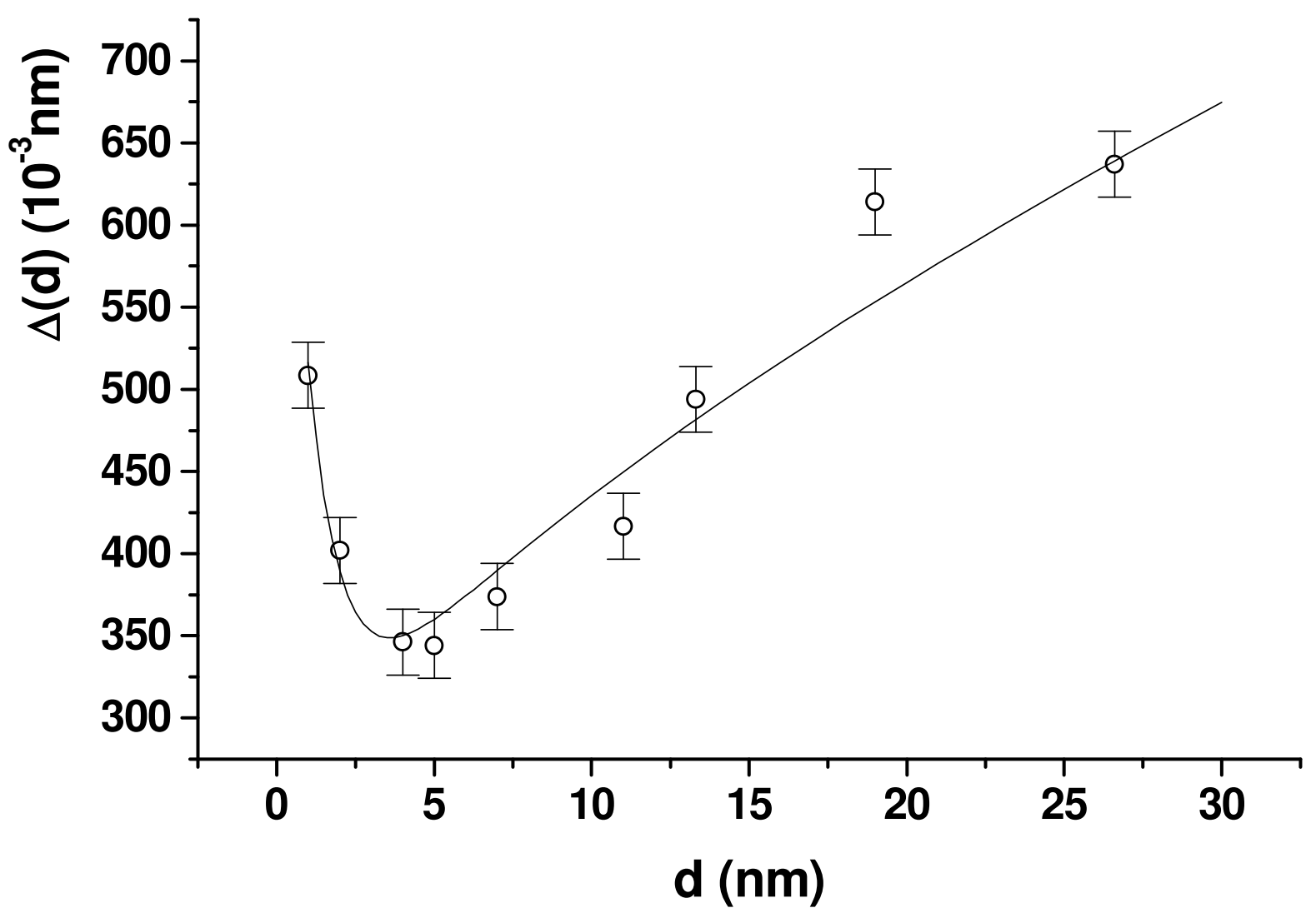

Figure 2: The roughness $\Delta_{\exp }(d)$ as a function of $d$. The experimental points are represented by open circles and the best fit values $\Delta(d)$, given by Eq.(1), by a solid line.

In Fig.(2) the open circles give the roughness of the platinum films $\Delta_{\exp }(d)$, measured by STM, as a function of $d$, obtained with a separated set of samples. The solid line represents the roughness $\Delta(d)$, obtained by a best fit of the experimental results, given by the equation:

$$
\Delta(d)=0.65 e^{-0.95 d}+0.22+0.045 d^{0.68} \text {. }
$$

The glass substrate roughness, measured by AFM, was found to be $\Delta_{\text {glass }}=(0.46 \pm 0.02) \mathrm{nm}$.

\section{Data Analysis and Conclusions}

In a recent paper[13]we have investigated the growing dynamics of Pt films, measuring the films roughness by STM technique. In this paper the films thickness goes from 10 up to $76 \mathrm{~nm}$. From the roughness, measured by STM, we determined the critical exponents, $\alpha$ and $\beta$, that are given by, $\alpha \approx 1$ and $\beta \approx 0.71 \pm 0.12$. We verified that, in agreement with the dynamical scaling laws of Family-Vicsek $[16,17]$ the roughness $\Delta$ and the lateral correlation length $\xi$ increase with the time deposition $\mathrm{t}$ according to the relations $\Delta \sim \mathrm{t}^{\beta} \sim d^{\beta}$ and $\xi \sim \mathrm{t}^{\beta / \alpha} \sim d^{\beta}$, since $d \sim \mathrm{t}$ and $\alpha \approx 1$. As well known[18], these relations are obeyed when films are deposited on substrates with negligible roughness or when the film thickness becomes much larger than the substrate roughness. For 
relatively small $d$, the substrate roughness plays the fundamental role in the film growing process and the scaling laws shown above, $\Delta \sim d^{\beta}$ and $\xi \sim d^{\beta}$, are not obeyed. In particular, $\Delta(d)$, seen in Fig.(2) has a peculiar behavior, decreasing in the interval $1.31 \mathrm{~nm} \leq d \leq 3 \mathrm{~nm}$, but it not possible to measure $\xi(d)$. This was done for thicker films, as shown in our recent work. ${ }^{14}$ So, according to preceding resistivity works[3,4,9], $\xi(d)$ will be determined in order to get a good agreement between theory and experimental data.

For $d \leq 3 \mathrm{~nm}$ the formation of valleys and hills in the Pt film is dictated mainly by the glass surface roughness, $\Delta_{\text {glass }} \approx 0.46 \mathrm{~nm}$. From Fig.(2) we see that only for $d \geq 2 \sim 3 \mathrm{~nm}$ the term $\mathrm{d}^{0.68}$ begins to contribute. This means that only for $d \geq 2 \sim 3 \mathrm{~nm}$ the substrate influence, in the film growing process, begins to decrease, being gradually substituted by a Pt growth dynamics, governed by a Kardar-Parisi-Zhang (KPZ) equation[13]. Note that $\beta$, here obtained by a best fit, is given by $\beta$ $=0.68$. This value is in good agreement, within the experimental errors, with $\beta=0.71 \pm 0.12$, determined in our precedent work[13]. The KPZ growing process will predominate only for $d>15$ $\mathrm{nm}$, when $\Delta(d)>\Delta_{\text {glass }} \approx 0.46 \mathrm{~nm}$. For $d<15 \mathrm{~nm}$ the film growing is essentially governed by the glass substrate roughness.

As pointed out before, many theoretical models, semiclassical and quantum mechanical, have been proposed[1-9] to explain the $\sigma(d)$ conductivity experimental results. Due to the progress in the technology of thin films deposition (metallic and semiconducting), recent experiments on the thin films electrical conductivity revealed more precise and detailed features in the transport properties[2-9]. These studies have clearly shown the occurrence of quantum size effects (QSE) in the $\sigma(d)$ when: (a) $d<\lambda_{0}$, where $\lambda_{0}$ is the electron mean free path, (b) the Fermi wavelength $\lambda_{\mathrm{F}}>\Delta$ and (c) the energy-level quantization is enhanced in the direction along the film thickness. This quantization is estimated by the parameter $\mathrm{N} \approx(3 \mathrm{n} / \pi)^{1 / 3} d$, which is the number of subbands occupied by the electron gas.

For platinum, $\lambda_{0} \approx 20 \mathrm{~nm}[19], \mathrm{n} \approx 6.6110^{28} / \mathrm{m}^{3}$, the Fermi wave vector $\mathrm{k}_{\mathrm{F}}=\left(3 \mathrm{n} \pi^{2}\right)^{1 / 3}=$ $12.51 \mathrm{~nm}^{-1}$ and $\lambda_{\mathrm{F}}=2 \pi / \mathrm{k}_{\mathrm{F}}=0.50 \mathrm{~nm}$.

For our films, $\Delta<0.5 \mathrm{~nm}$ and $d$ are in the interval $1.31 \leq d \leq 11.66 \mathrm{~nm}$. This implies that $\mathrm{N}$ is in the range $5 \leq \mathrm{N}<48$. So, as the above mentioned conditions are obeyed, we verify that QSE are expected to be dominant in the electron conductivity for the Pt films. So, we will compare our experimental results with theoretical predictions obtained with a quantum mechanical formalism.

Let us consider, for instance, the quantum mechanical approach developed by Fishman and Calecki[3,4]. They have assumed that the film resistivity $\rho$ is due to electronic scattering produced only by bulk properties $\left(\rho_{\text {bulk }}\right)$ and film surface roughness $\left(\rho_{\mathrm{s}}\right)$, that is, $\rho=\rho_{\text {bulk }}+\rho_{\mathrm{s}}$. According to their calculations, in the limiting conditions $\mathrm{N} \gg>1$ and $\mathrm{k}_{\mathrm{F}} \xi<<1$, the conductivity $\sigma_{\mathrm{s}}(d)$ due the surface roughness is given by:

$$
\sigma_{s}(d)=\frac{3^{-1 / 3}}{4 \pi^{3 / 2}} \frac{e^{2}}{\hbar} \frac{n^{-1 / 3} d^{2}}{(\Delta \xi)^{2} F(0)}\left[1-\frac{6}{d\left(3 n \pi^{5}\right)^{-1 / 3}}\right]
$$

where $e$ is the electron charge, $\hbar$ the Planck constant, $n$ the electronic density and $\Delta(d)$ the surface roughness; $\mathrm{F}(0)$, calculated for $\mathrm{k}_{\mathrm{F}} \xi<1$, is a function which takes into account correlation effects on the film surfaces. It depends on the model adopted to estimate the correlation effects. We will put $\mathrm{F}(0)=\pi$, according to the Gaussian model.

Considering $d, \Delta$ and $\xi$ in $\mathrm{nm}$ and putting $\mathrm{n} \approx 6.6110^{28} / \mathrm{m}^{3}$ in Eq. (2), we see that $\rho(d) / \rho_{\text {bulk }}=$ $1+\rho_{\mathrm{s}}(d) / \rho_{\text {bulk }}$, taking $\rho_{\text {bulk }}=1.0610^{-7} \Omega \mathrm{m}$, is given by :

$$
\rho(d) / \rho_{\text {bulk }}=1+6084.17(\Delta \xi)^{2} /\left[d^{2}(1-0.153 / d)\right] \text {. }
$$


Now, using Eq.(3), $\xi$ will be determined to obtain, at $d=1.31 \mathrm{~nm}, \rho(d) / \rho_{\text {bulk }}=\rho_{\text {exp }} / \rho_{\text {bulk }} \approx 20$, with $\Delta(1.31)=0.46 \mathrm{~nm}$, given by Eq.(1). We verified that the adjusted $\xi$ is given $\xi=0.143 \mathrm{~nm}$. This value is smaller than the Pt lattice constant $(\sim 0.4 \mathrm{~nm})$. This also occurs with the $\mathrm{CoSi}_{2}$ thin films, according to Fishman and Calecki, ${ }^{3,4}$ and Palasantzas et al[9].

So, for $d \leq 1.8 \mathrm{~nm}, \xi(d)$ will be taken as $\xi=$ constant $\approx 0.143 \mathrm{~nm}$. On the other side, since for $d \geq 1.8 \mathrm{~nm}$, $\xi$ is expected to be roughly estimated by $\xi(d) \sim \mathrm{d}^{0.68}$, we will assume that,

$$
\xi(d)=0.109 d^{0.68}-0.145 e^{(-1.2 d)}
$$

This function $\xi(d)$ was written in order to connect $\xi=0.143 \mathrm{~nm}$, for $d \leq 1.8 \mathrm{~nm}$, and $\xi(d) \sim$ $\mathrm{d}^{0.68}$, for $d \geq 1.8 \mathrm{~nm}$. Our approach permits us to understand more clearly the lateral correlation effects. Indeed, for very thin films, that is, for $d<2 \mathrm{~nm}, \xi$ assumes a minimum value due to the influence of the glass substrate which strongly inhibits the correlation effect between the Pt atoms. As the Pt film thickness increases, the substrate influence gradually decreases. So, for $d>2 \mathrm{~nm}$, the correlation length $\xi$ begins to increase as $\xi(d) \sim d^{0.68}$, according to the Family-Vicsek [17] relations and a KPZ equation[13-15].

Substituting $\Delta(d)$ and $\xi(d)$, given by Eqs.(1) and (4), into Eq.(3) we calculate $\rho(d) / \rho_{\text {bulk. }}$. In Fig.(1) are shown, for $1.31 \leq d \leq 11.66 \mathrm{~nm}$, the predicted values, indicated by a solid line, and the experimental data $\rho_{\text {exp }}(d) / \rho_{\text {bulk }}$, designated by circles. We see that a good agreement is found between theory and experiment.

For $d<2 \mathrm{~nm}, \sigma_{\mathrm{s}}(d)$ obeys the exponential law $\sigma_{\mathrm{s}}(d) \sim d^{2.9}$. For $2 \leq d \leq 11.66 \mathrm{~nm}$, we verify that $\sigma_{\mathrm{s}}(d)$ cannot be written as $\sigma_{\mathrm{s}}(d) \sim \mathrm{d}^{\mathrm{s}}$. Only for thicker films $(d>20 \mathrm{~nm})$ the conductivity will be described by a linear law $\sigma_{\mathrm{s}}(d) \sim d$, according to classical approaches[1,5,20,21].

\section{Summary}

We fabricated platinum thin films by vacuum arc plasma deposition with thickness $d$ going from 1.31 to $11.66 \mathrm{~nm}$ and measured their resistivity as a function of $d$. Our resistivity results are quite similar to those found by Avrekh et al.[15] for Pt thin films, obtained with the same deposition process, with thickness going from 2.6 up to $19 \mathrm{~nm}$. We have also measured the surface roughness and determined the surface correlation length of the films, as a function of $d$. We compared our resistivity results with theoretical predictions obtained with the quantum mechanical formalism proposed by Fishman and Calecki[3,4]. A good agreement is found between the resistivity experimental data and theoretical predictions.

\section{$\underline{\text { Acknowledgements }}$}

The authors thank FAPESP, CAPES and the CNPq for the financial support.

\section{$\underline{\text { References }}$}

[1] C.R. Tellier, A.J. Tosser, "Size Effects in Thin Films", Elsevier Scientific Publishing Company (1982).

[2] Z. Tesanovic, M.V. Jaric, S. Maekawa, Phys. Rev. Lett. 57, 2760 (1986).

[3] G. Fishman, D. Calecki, Phys. Rev. Lett. 62, 1302 (1989).

[4] G. Fishman, D. Calecki, Phys. Rev. B43, 11581 (1991).

[5] M. Jalochowski, E. Bauer, H. Knoppe, G. Lilienkamp, Phys. Rev. B45, 13607 (1992).

[6] M. Jalochowski, M. Hoffman, E. Bauer, Phys. Rev. Lett. 76, 4227 (1996).

[7] G. Palasantzas, J. Barnas, Phys. Rev. E56, 7726 (1997). 
[8] G. Palasantzas, Phys. Rev. B58, 9685 (1998).

[9] G. Palasantzas, Y.P. Zhao, G.C. Wang, T.M. Lu, J. Barnas and J.Th.M. de Hosson .Phys. Rev. B61, 11109 (2000).

[10] I.G. Brown, A. Anders, M.R. Dickinson, R.A. MacGill, O.R. Monteiro, Surf. Coat. Technol. $\underline{112}, 271$ (1999).

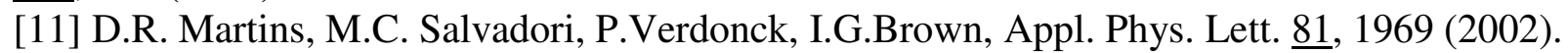

[12] M.C. Salvadori, I.G. Brown, A. R. Vaz, L.L. Melo, M. Cattani - "Measurement of the Elastic Modulus of Nanostructured Gold and Platinum Thin Films", Phys. Rev. B (2003). To be published.

[13] M.C. Salvadori, L.L. Melo, M. Cattani, O.R. Monteiro and I.G. Brown, Surf. Rev. Lett. 10, 1 (2003).

[14] M. Cattani, M.C. Salvadori, Surf. Rev. Lett. $\underline{8}, 347$ (2001).

[15] M. Avrekh, O.R. Monteiro, I.G. Brown, Applied Surface Science 158, 217 (2000).

[16] A.L. Barabasi, H.E. Stanley, "Fractal Concepts in Surface Growth", Cambridge University Press (1995).

[17] F. Family, T. Vicsek, J. Phys. A18, L75 (1985).

[18] T.J. Silva, J.G. Moreira, Phys. Rev. E56, 4880 (1997).

[19] C. Kittel, "Introduction to Solid State Physics", J. Wiley, New York (1976).

[20] C.K. Ghosh and A.K. Pal, J. Appl. Phys. 51(4), 2281 (1980).

[21] J.C. Hensel, R.T. Tung, J.M. Poate and F.C. Unterwald, Phys. Rev. Lett. 54, 1840 (1980). 